\title{
Acute effects of trauma-focused research procedures on participant safety and distress
}

\author{
Vanessa M. Brown ${ }^{a, b}$, Jennifer L. Strauss ${ }^{a, c, d}$, Kevin S. LaBar ${ }^{b, c}$, Andrea L. Gold ${ }^{e}$, \\ Gregory McCarthy ${ }^{\mathrm{e}}$, Rajendra A. Morey ${ }^{\mathrm{a}, \mathrm{b}, \mathrm{c}, \mathrm{*}}$ \\ a Mental Illness Research Education and Clinical Center for Post Deployment Mental Health, Durham VA Medical Center, Durham, NC, USA \\ ${ }^{\mathrm{b}}$ Duke-UNC Brain Imaging and Analysis Center, Duke University, Durham, NC, USA \\ ${ }^{\mathrm{c}}$ Department of Psychiatry and Behavioral Sciences, Duke University, Durham, NC, USA \\ ${ }^{\mathrm{d}}$ Center for Health Services Research in Primary Care, Durham, NC, USA \\ e Department of Psychology, Yale University, New Haven, CT, USA
}

\section{A R T I C L E I N F O}

\section{Article history:}

Received 15 February 2013

Received in revised form

29 October 2013

Accepted 31 October 2013

Available online 8 November 2013

\section{Keywords:}

PTSD

Trauma

Research risks

Risk assessment

Suicidal ideation

Homicidal ideation

Distress

\begin{abstract}
A B S T R A C T
The ethical conduct of research on posttraumatic stress disorder (PTSD) requires assessing the risks to study participants. Some previous findings suggest that patients with PTSD report higher distress compared to non-PTSD participants after trauma-focused research. However, the impact of study participation on participant risk, such as suicidal/homicidal ideation and increased desire to use drugs or alcohol, has not been adequately investigated. Furthermore, systematic evaluation of distress using pre- and post-study assessments, and the effects of study procedures involving exposure to aversive stimuli, are lacking. Individuals with a history of PTSD $(n=68)$ and trauma-exposed non-PTSD controls $(n=68)$ responded to five questions about risk and distress before and after participating in research procedures including a PTSD diagnostic interview and a behavioral task with aversive stimuli consisting of mild electrical shock. The desire to use alcohol or drugs increased modestly with study participation among the subgroup $(n=48)$ of participants with current PTSD. Participation in these research procedures was not associated with increased distress or participant risk, nor did study participation interact with lifetime PTSD diagnosis. These results suggest some increase in distress with active PTSD but a participant risk profile that supports a favorable risk-benefit ratio for conducting research in individuals with PTSD.
\end{abstract}

Published by Elsevier Ireland Ltd.

\section{Introduction}

Conducting research with traumatized individuals poses an ethical challenge - such research seeks to improve the lives of trauma survivors, while simultaneously asking participants to recollect painful trauma experiences or recount their trauma narratives. Consequently, trauma-focused research involves the risk of producing distress and possible exacerbation of symptoms among participants (Newman and Kaloupek, 2009). The paramount ethical concern is ensuring that study procedures do not precipitate acute symptom changes, leading to serious thoughts of harming oneself or others. Such grave concerns for the safety of participants generally eclipse other risks typically associated with trauma-focused research. Previous studies examining distress in trauma-exposed participants have found that elevated distress is

\footnotetext{
* Correspondence to: Department of Psychiatry, 2424 Erwin Road, Suite 501, Durham, NC 27705, USA. Tel.: +1 919286 0411x6425; fax: +1 9194165912.

E-mail addresses: rajendra.morey@gmail.com, rajendra.morey@duke.edu (R.A. Morey).
}

associated with recounting traumatic experiences, more severe symptoms, greater trauma exposure, or diagnosis of PTSD (Walker et al., 1997; Parslow et al., 2000; Deprince and Chu, 2008). These findings have led to ethical concerns about the effects of study participation among trauma-exposed individuals. However, traumatized participants consistently report a lack of regret of study participation at the conclusion of studies, even when they rate the study material distressing. Participants also report a willingness to enroll in similar studies in the future (Griffin et al., 2003; Cromer et al., 2006; Deprince and Chu, 2008) Therefore, the source of distress remains unclear.

Prior studies have examined distress (e.g., dislike of study procedures or regret about participation) and perceived benefits directly related to participation (Walker et al., 1997; Parslow et al., 2000; Cromer et al., 2006; Deprince and Chu, 2008; Resick et al., 2009) but have not examined participants' potential for harm to self or others. In their study, Cromer et al. (2006) found that undergraduates rated trauma-related questionnaires no more distressing, compared to everyday events, than other questionnaires about academic achievement, body image, parents' income, 
race, and sexuality. The students viewed trauma-related information as more important and possessing greater cost-benefit ratio than other questions. Deprince and Chu (2008) used the Reactions to Research Participation Questionnaire in undergraduate and community samples to find low ratings on the drawbacks scale and high ratings on scales measuring personal benefit, global evaluation, and participation, which indicated a positive study experience. Walker et al. (1997) found that a community sample rated study participation as a positive experience, but participants with a history of abuse rated the materials as more upsetting. In a military veteran sample, Parslow et al. (2000) found frequent reports of distress among people with a history of PTSD, which were unrelated to willingness to participate in future studies or an increased utilization of medical services. Resick et al. (2009) found that participants with PTSD rated study procedures with traumarelated material to be distressing but more interesting. Greater perceived burden of assessments at pretreatment predicted greater chance of treatment completion. These participants also reported experiencing strong emotions during participation, but most rated the study materials as interesting and not distressing (Griffin et al., 2003). To accurately make risk-benefit judgments in traumatized individuals (Newman and Kaloupek, 2009), the impact of study participation on homicidal and suicidal ideation needs to be assessed empirically. Thus, our primary goal was to study the effect of trauma-focused research procedures on clinical distress and potential for harm (e.g. suicidal ideation) in patients with PTSD.

A major source of uncertainty about the source of distress stems from the lack of pre- vs. post-study distress assessment, making it unclear whether participant distress is due to study procedures or other pre-existing factors. Pre- vs. post-study assessment in other at-risk populations showed decreased distress in borderline personality disordered patients with suicidality (Reynolds et al., 2006), but no change in recent HIV seroconverters (Scarvalone et al., 1996). Pre- vs. post-participation assessment of a traumatized population found that participants with PTSD reported a greater increase in sadness and tension from completing trauma-related questionnaires than from trauma-unrelated questionnaires (FerrierAuerbach et al., 2009). In addition, it remains unclear whether participants with PTSD are more distressed by study procedures than trauma-exposed participants without PTSD. The literature has rarely examined whether the effects of PTSD persist after remission, despite high rates of relapse (Solomon and Mikulincer, 2006). Veterans with current or past PTSD reported greater distress when asked about traumatic experiences than those who had not developed PTSD (Parslow et al., 2000). Thus, our secondary goal was to use pre- vs. post-study measures to ascertain distress associated with participation in trauma-focused study procedures (diagnostic interview) and exposure to aversive stimuli (mild electrical shock) in individuals with PTSD.

Based on previous findings of increased distress among participants with PTSD after trauma-related study procedures (Griffin et al., 2003; Ferrier-Auerbach et al., 2009), we hypothesized that participants with a history of PTSD would show increased concerns of clinical distress and potential for harm after undergoing trauma-focused diagnostic interview, but the addition of trauma-unrelated aversive stimuli would not increase this risk. We assessed risk by questioning the participants about thoughts of suicide, self-harm, harm to others, drug or alcohol use, and stress level. Potential for harm and clinical distress were assessed in traumatized individuals, with and without PTSD, before and after administration of a diagnostic interview for PTSD, and exposure to trauma-unrelated aversive stimuli consisting of mild electrical shocks.

\section{Methods}

\subsection{Participants}

Veterans enrolled in a post-deployment mental health registry of United States military personnel who served after September 11, 2001 (Dedert et al., 2009) were contacted by telephone for a series of studies on fear processing in PTSD. Participants were free from psychiatric disorders other than PTSD, major depression, and past substance abuse based on Structured Clinical Interview for DSM-IV (SCID) (First et al., 1997) evaluation. Of the 136 participants (see Table 1 for clinical and demographic data), 73 (54\%) were Caucasian, 54 (40\%) were African-American, and $9(7 \%)$ were of other races. The majority of participants had experienced combat or a war zone as their primary trauma $(n=95,70 \%)$; other traumas experienced were sudden deaths $(n=11,8 \%)$, sexual assaults $(n=6,4 \%)$, transportation accidents $(n=6,4 \%)$, childhood abuse $(n=5,4 \%)$, or other forms of trauma $(n=13,10 \%)$. Five additional participants who were missing data for the post-study time point were excluded, four withdrew because of contraindication to magnetic resonance imaging (MRI), and one withdrew due to discomfort during the diagnostic interview. Participants provided informed consent to procedures approved by the Durham VA Medical Center and Duke University Health System Institutional Review Boards (IRBs). Participants were compensated $\$ 25 / \mathrm{h}$ plus travel costs.

\subsection{Measurements}

All participants completed the Clinician Administered PTSD Scale (CAPS) (Blake et al., 1995), a diagnostic structured clinical interview for current and lifetime PTSD symptoms and completed questionnaires about mental health and trauma exposure. For clinical characterization, all participants completed the Beck Depression Inventory (BDI) to obtain a continuous measure of depressive symptoms (Beck et al., 1988). As BDI scores were highly correlated with PTSD symptoms, the analyses reported focus on PTSD rather than depressive symptoms. Scores from the Alcohol Use Disorders Test (AUDIT (Saunders et al., 1993)) that is designed to assess recent misuse of elicit substances and the substance use disorders diagnoses from the SCID for secondary analyses concerning substance use urges, were obtained from the post-deployment registry (Dedert et al., 2009).

\subsection{Procedures}

We included data from behavioral and neuroimaging paradigms designed to understand fear processing in PTSD. As a part of study procedures, approximately half of the participants ( $n=64,47 \%$ ) received $12-16$ mild electrical shocks delivered to the upper (wrist) or lower (ankle) extremity in a behavioral testing room or MRI scanner suite. The intensity (voltage) of shocks was determined on an individual basis prior to the start of each study by increasing intensity in $10-\mathrm{V}$ increments (maximum of 100V) until the participant deemed the shock as "annoying but not painful." The balance of participants $(n=72,53 \%)$ underwent an MRI scan without

Table 1

Participant demographic and clinical data.

\begin{tabular}{|c|c|c|c|}
\hline Clinical Measure & Control $(n=68)$ & $\operatorname{PTSD}(n=68)$ & Comparison \\
\hline Age, mean (S.D.) & $40.3(11.2)$ & $39.1(8.9)$ & $t(134)=0.71 ; p>0.4$ \\
\hline Gender, number (\%) of women & $6(8.8)$ & $13(19.1)$ & $\chi^{2}(1)=3.0, p>0.05$ \\
\hline CAPS, mean (S.D.) & $8.59(10.2)$ & $57.0(27.7)$ & $t(134)=13.5 ; p<0.001$ \\
\hline BDI, mean (S.D.) & $4.6(6.1)$ & $18.6(14.9)^{a}$ & $t(133)=7.2 ; p<0.001$ \\
\hline AUDIT, mean (S.D.) & $2.8(2.5)$ & $4.4(5.2)$ & $t(134)=2.3 ; p<0.05$ \\
\hline Depression diagnosis, number (\%) & $12(18)$ & $48(71)$ & $\chi^{2}(1)=38.7 ; p<0.001$ \\
\hline Substance use diagnosis, number (\%) & $14(21)$ & $12(18)$ & $\chi^{2}(1)=2.8, p>0.05$ \\
\hline
\end{tabular}

CAPS=Clinician Administered PTSD Scale; $\mathrm{BDI}=$ Beck Depression Inventory; AUDIT=Alcohol Use Disorders Test

$$
\text { a } n=67 \text {. }
$$


the electrical shock. One of four study coordinators involved in the study administered all procedures to each participant, which lasted approximately $4 \mathrm{~h}$.

\subsection{Measures of clinical distress and potential for harm}

Participants provided verbal ratings on a Likert scale (1-7) to indicate their suicidal urges, urge to harm self, urge to harm others, urge to use drugs or alcohol, and level of stress. Study coordinators administered the questionnaire for assessing clinical distress and potential for harm based on the University of Washington Risk Assessment Protocol (Reynolds et al., 2006) prior to initiating study procedures and repeated the questionnaire immediately after study procedures were completed. Clinical evaluation by a board-certified psychiatrist (RAM) was initiated for any participants that exceeded the following predetermined criteria for level of stress $(>3)$, self-harm urges $(>2)$, suicidal urges $(>1)$, substance use urges $(>3)$, and

Table 2

Pre- and post-participation levels of distress by PTSD diagnosis.

\begin{tabular}{|c|c|c|c|c|c|c|}
\hline \multirow[t]{2}{*}{ Diagnosis } & \multirow{2}{*}{$\begin{array}{l}\text { Safety/distress } \\
\text { measure }\end{array}$} & \multirow[t]{2}{*}{ Group } & \multicolumn{2}{|c|}{ Pre-study } & \multicolumn{2}{|c|}{ Post-study } \\
\hline & & & Mean & S.D. & Mean & S.D. \\
\hline \multirow[t]{10}{*}{ A. Lifetime PTSD } & \multirow[t]{2}{*}{ Stress } & Control $(n=68)$ & 1.89 & 1.18 & 1.73 & 1.32 \\
\hline & & PTSD $(n=68)$ & 3.17 & 1.67 & 3.02 & 1.74 \\
\hline & \multirow[t]{2}{*}{ Self-harm } & Control & 1 & 0 & 1 & 0 \\
\hline & & PTSD & 1.06 & 0.17 & 1.03 & 0.17 \\
\hline & \multirow[t]{2}{*}{ Suicidality } & Control & 1 & 0 & 1 & 0 \\
\hline & & PTSD & 1.02 & 0.12 & 1.03 & 0.17 \\
\hline & \multirow[t]{2}{*}{ Substance Use } & Control & 1.13 & 0.52 & 1.13 & 0.67 \\
\hline & & PTSD & 1.28 & 0.73 & 1.52 & 1.31 \\
\hline & \multirow[t]{2}{*}{ Harm to Others } & Control & 1.03 & 0.24 & 1.02 & 0.12 \\
\hline & & PTSD & 1.21 & 0.61 & 1.27 & 0.68 \\
\hline \multirow[t]{10}{*}{ B. Current PTSD } & \multirow[t]{2}{*}{ Stress } & Control $(n=68)$ & 1.89 & 1.18 & 1.73 & 1.32 \\
\hline & & PTSD $(n=48)$ & 3.62 & 1.67 & 3.5 & 1.77 \\
\hline & \multirow[t]{2}{*}{ Self-harm } & Control & 1 & 0 & 1 & 0 \\
\hline & & PTSD & 1.08 & 0.28 & 1.04 & 0.2 \\
\hline & \multirow[t]{2}{*}{ Suicidality } & Control & 1 & 0 & 1 & 0 \\
\hline & & PTSD & 1.02 & 0.14 & 1.04 & 0.2 \\
\hline & \multirow[t]{2}{*}{ Substance Use } & Control & 1.13 & 0.52 & 1.13 & 0.67 \\
\hline & & PTSD & 1.31 & 0.75 & 1.72 & 1.52 \\
\hline & \multirow[t]{2}{*}{ Harm to Others } & Control & 1.03 & 0.24 & 1.02 & 0.12 \\
\hline & & PTSD & 1.27 & 0.71 & 1.34 & 0.75 \\
\hline
\end{tabular}

urge to harm others $(>2$ ). All 49 participants who were clinically evaluated (36 with current PTSD, 2 lifetime PTSD, and 11 trauma-exposed controls) were determined to be clinically stable and discharged home without further clinical intervention after completing the remainder of study procedures.

\subsection{Statistical analyses}

Repeated measures analysis of covariance (ANCOVA) was performed for each of the five measures. Pre- and post-study ratings served as the dependent repeatedmeasures. Categorical variables were based on PTSD diagnosis (lifetime PTSD analyses: lifetime PTSD vs. Control; current PTSD analyses: current PTSD vs. Control) and exposure to the aversive stimuli (trauma-focused exposure vs trauma-focused + aversive stimulus exposure). The identity of the study coordinator was entered as a covariate of no interest. Despite lack of current symptoms patients with lifetime PTSD were deemed to be at risk for experiencing clinical distress and potential for harm, and therefore were included in the primary lifetime PTSD analyses. Secondarily, current PTSD analyses contrasted patients with current PTSD to controls given that current symptoms may constitute elevated risk for exacerbation as compared to past symptoms. For significant differences $(p<0.05)$ the magnitude of means were compared to determine the direction of the result.

\section{Results}

\subsection{Lifetime PTSD}

As assessed by the CAPS interview, 68 participants had a diagnosis of either current $(n=48)$ or past $(n=20)$ PTSD and 68 were in the trauma-exposed control group. Of the $64(47 \%)$ participants who received mild electrical shocks, 22 (34\%) had current PTSD, 8 (13\%) had past PTSD, and 34 (53\%) were traumaexposed controls. The means and standard deviations of all preand post-study clinical distress and potential for harm measures are reported in Table 2 and Fig. 1 . The primary analyses found a main effect of PTSD diagnosis for stress $\left(F_{1,131}=33.46, p<0.001\right)$, substance use urges $\left(F_{1,131}=4.12, p=0.042\right)$, and urges to harm others $\left(F_{1,131}=6.63, p=0.011\right)$. Participants with PTSD reported higher levels of all three measures. The main effects and all interactions with time (pre- vs. post-study), as well as the effect of aversive stimulus exposure were non-significant.

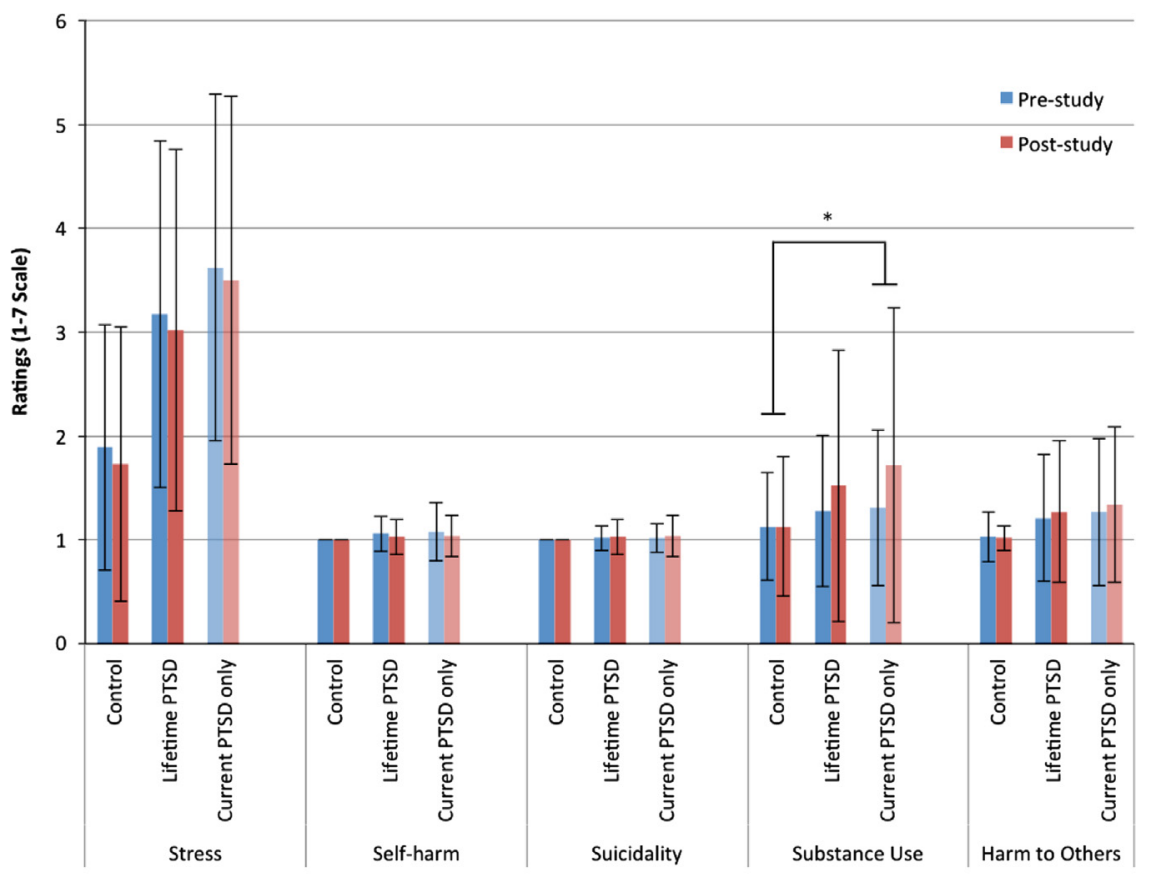

* significant $(p<.05)$ time by diagnosis interaction

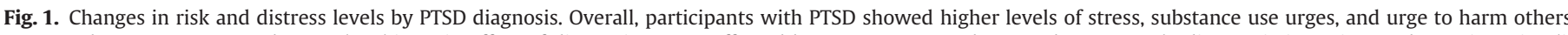

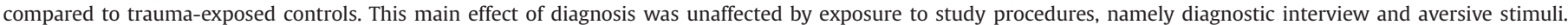
Substance use urges were aggravated by study procedures, but only in patients with current PTSD $\left(F_{1,111}=5.93, p=0.016\right)$. 


\subsection{Current PTSD}

The secondary analysis, comparing current PTSD to traumaexposed controls, found a similar main effect of PTSD diagnosis for stress $\left(F_{1,111}=52.52, p<0.001\right)$, substance use urges $\left(F_{1,111}=6.56\right.$, $p=0.012)$, and urges to harm others $\left(F_{1,111}=8.285, p=0.005\right)$, with higher ratings in the PTSD group than the control group. Substance use urges showed a significant main effect of time $\left(F_{1,111}=4.92\right.$, $p=0.029$ ) with higher post-study ratings compared to pre-study ratings, and a significant interaction of time by PTSD diagnosis $\left(F_{1,111}=5.93, p=0.016\right)$, such that patients with current PTSD reported larger increases in substance use urges than controls. Current PTSD status had no interaction of time by presence of electrical shocks. The main effect of time (pre- vs. post-study) and the interaction of time-by-diagnosis on substance use urges remained significant after including scores from the AUDIT $\left(F_{1,110}=6.59, p=0.012\right)$ or diagnostic status $\left(F_{1,110}=5.37, p=0.022\right)$ for substance use disorders as covariates.

\section{Discussion}

In the current study, we examined whether participating in a study with trauma-focused study procedures (diagnostic interview) and exposure to aversive stimuli (mild electrical shock) resulted in increased concerns of clinical distress or potential for harm (e.g. suicidal ideation) in individuals with PTSD. Pre-study measurements found that participants with PTSD showed higher levels of stress, substance use urges, and urge to harm others compared to trauma-exposed controls. Increased ratings were present among all participants with a history of PTSD as well as those with current PTSD only. This main effect of diagnosis was not altered by exposure to study procedures, namely diagnostic interview and electrical shock. Substance use urges were aggravated by study procedures, but only in patients with current PTSD, and only to a modest extent (less than a half-point on a 7-point scale). Thus, this change constituted the only negative impact resulting from study procedures, but its magnitude was not deemed clinically significant. Clinical distress and potential for harm were unaffected by the participants' exposure to mild electrical shock.

Our findings add an important dimension to understanding research-related clinical distress or potential for harm in traumatized participants. Other than increased substance use urges, the lack of increase in safety-related concerns with study participation was consistent with prior studies examining other dimensions of distress associated with research participation in traumatized participants (Griffin et al., 2003; Cromer et al., 2006; Deprince and Chu, 2008). The elevated levels of stress, substance use urges, and urges to harm others observed in patients with PTSD highlights the importance of isolating baseline levels of distress in a psychiatric population from safety-related concerns and distress resulting directly from study procedures. Safety ratings were not influenced by exposure to a stressful behavioral paradigm that included mild electrical shock. Through informal debriefing of participants, it appears there was greater negative impact of these procedures from the anticipatory anxiety associated with impending shock, than from the discomfort or pain imparted by stimulus delivery. Although Ferrier-Auerbach et al. (2009) found traumarelated material to be distressing, the relative effects of stressful procedures versus exposure to trauma-related material remained unclear in their findings. Meanwhile, Griffin et al. (2003) found recounting traumatic experiences more distressing for participants with PTSD than those without PTSD, an effect that was not found with trauma-unrelated material.
By examining clinical distress and potential for harm, our study offers a more complete appraisal of the risk-benefit ratio for research on traumatized participants than previous work. Previous studies have found that traumatized individuals report research participation accrued personal benefit, and benefit to science and society (Cromer et al., 2006; Deprince and Chu, 2008), but that increased distress can still occur (Ferrier-Auerbach et al., 2009). The novel contribution of our study was that potential for harm such as homicidal and suicidal ideation does not appear to be affected by research participation, contrary to the perceptions of some in the trauma research community and members of IRBs (Cromer et al., 2006; Ferrier-Auerbach et al., 2009).

However, the small increase in the desire to use drugs or alcohol in patients with current PTSD highlights an important concern. Substance use is associated with its own set of concerns including the possibility of overdose and engaging in risky behavior. Our analysis shows that this increase was not accounted for by levels of prior alcohol use or abuse, but may be due to other unexplored factors. For instance, substance use urges might suggest an attempt to cope with a component of distress that was not captured by our questions. Alternatively, participants may not be consciously aware of elevated stress, and therefore unaware that this "hidden" stress has precipitated an increase in desire to use substances. Thus, the urge to use substances may reflect a coping mechanism. A straightforward explanation for increased substance use urges may reflect simply a time-of-day effect, given that most participants completed the post-study questionnaire in the late afternoon while the pre-study questionnaire was completed in the morning. Alternatively, the long duration and/or hospital location of the testing procedures alone may have contributed to these urges. The interaction with current PTSD shows that this effect was more prominent in those with current PTSD, possibly related to higher rates of comorbid substance use in this group. For participants who misuse alcohol, restricted access to drinking for several hours (during study procedures) may contribute to increased post-study urges to drink. Moreover, while statistically significant, the increase in substance use urges was less than half a point on a seven-point scale. It is unclear whether this low magnitude increase in desire to use substances would produce a clinically significant increase in substance use, i.e. result in functional impairment. Therefore, the increased desire to use substances requires further study.

Whether study procedures dealing with trauma are more distressing than trauma-unrelated procedures may depend on the population and the specific research procedure in question. For example, in a nonclinical sample of college students, Cromer et al. (2006) found that participants did not perceive greater distress from trauma-related questions than from other personal questions about topics such as weight and academic achievement. Participants with PTSD rated questionnaires about trauma as less stressful, and diagnostic interviews more stressful, than a psychophysiological task with trauma-unrelated stressors (Resick et al., 2009). One the other hand, participants with PTSD also report greater distress after completing trauma-related vs. unrelated questionnaires (Ferrier-Auerbach et al., 2009).

Despite subjective ratings of trauma-focused research participation as negative or distressing, traumatized participants have perceived participation as personally beneficial (Ferrier-Auerbach et al., 2009). For example, even when research participants have reported increased sadness and tension following administration of both trauma-related and unrelated questionnaires, they have endorsed participation as personally beneficial and were undeterred from future participation. Cromer et al. (2006) did not find increased distress associated with trauma history questionnaires in a non-clinical population, and "severely mentally ill" participants with PTSD rated trauma-related study questions as more 
beneficial than trauma-unrelated study questions (Grubaugh et al., 2012). These results suggest that participants are able to differentiate perceived long-term benefits of research participation from any acute negative impacts.

\subsection{Limitations}

Our study design does not account for anticipatory anxiety that participants may have felt at the beginning of the study. This anxiety derived from uncertainty or lack of knowledge about study details, may have inflated pre-study ratings. This phenomenon is analogous to the so-called "white-coat effect" where patient visits to the physician or other healthcare providers, particularly new encounters, are associated with elevated anxiety (Gerin et al., 2006). Given that participants in the present study had already taken part in a previous study (post-deployment registry) at our Center, their willingness to participate in the present study may represent self-selection of individuals who are less distressed by study procedures. This investigation did not find an effect of a procedure involving aversive stimuli (mild electrical shock) on participant distress. However, participants also underwent an MRI scan, which may have been perceived as aversive. Given the low baseline rate of suicidal and homicidal thoughts, even in our traumatized and chronically mentally ill samples, inadequate power may have limited our ability to find effects of study procedures on suicidal and homicidal ideation. Our use of single item question to assess each area of distress may have some inherent lack of reliability. Currently, no measure exists to investigate safety and distress associated with study participation more thoroughly. The development and use of such a questionnaire would provide more in-depth information about reactions to research participation. The study lacked a neutral control condition matched for duration and location and did not vary time-of-day explicitly. Our data could not conclusively inform whether it is the nature of the trauma interview itself that leads to increased clinical distress/safety concerns or whether a stressful procedure of any sort leads to increased clinical distress/safety concerns. Investigating a serial order effect would require independent distress measurements associated with exposure to aversive stimuli. Thus, the lack of a design that permits investigating such an effect represents a limitation of our study. On the other hand, our third analysis (trauma-focused exposure vs. traumafocused + aversive stimulus exposure) provides valuable information if interpreted with these caveats. Furthermore, our study was limited by lack of information about the long-term effects of study participation. Future studies should examine the risks and benefits of research over a longer period than one day of study. Response bias may have affected ratings in participants who felt uncomfortable disclosing their feelings to the study coordinator. Responses may have been influenced by the participants' desire to please the investigator with answers that met the perceived expectations of the interviewer. Future research should investigate these issues.

\subsection{Conclusion}

Our results indicate that overall concerns about risk or distress among patients with PTSD were not exacerbated by study participation, which runs counter to many prevailing assumptions about the risks of trauma-focused research (DePrince and Freyd. 2004). It is important to note that the present data represents group means and therefore individual participants may deviate from these means. As such, a comprehensive safety plan for careful assessment and intervention is an essential part of every research protocol involving traumatized populations. In addition, potential risks from study participation must be balanced against the need for a better understanding of PTSD (i.e., beneficence) through the conduct of ethically-informed PTSD research. In sum, these findings support a favorable risk-benefit ratio in conducting research with traumatized individuals with PTSD but raise concerns about possible increased desire to use substances - perhaps related to time-of-day or test duration effects - which require further investigation.

\section{Acknowledgment}

This research was supported by the National Institutes of Health Grant K23 MH073091 (RAM), Department of Veteran Health Affairs Career Development Award (JLS), VA Merit Grant 00507153 (RAM, VMB), Mental Illness Research Education and Clinical Center Grant for Post-Deployment Mental Health (RAM, JLS, GM)

\section{References}

Beck, A.T., Steer, R.A., Garbin, M.G., 1988. Psychometric properties of the beck depression inventory: twenty-five years of evaluation. Clinical Psychology Review 8, 77-100.

Blake, D.D., Weathers, F.W., Nagy, L.M., Kaloupek, D.G., Gusman, F.D., Charney, D.S. Keane, T.M., 1995. The development of a clinician-administered PTSD scale. Journal of Traumatic Stress 8, 75-90.

Cromer, L.D., Freyd, J.J., Binder, A.K., DePrince, A.P., Becker-Blease, K., 2006. What's the risk in asking? Participant reaction to trauma history questions compared with reaction to other personal questions. Ethics \& Behavior 16, 347-362.

Dedert, E.A., Green, K.T., Calhoun, P.S., Yoash-Gantz, R., Taber, K.H., Mumford, M.M. Tupler, L.A., Morey, R.A., Marx, C.E., Weiner, R.D., Beckham, J.C., 2009. Association of trauma exposure with psychiatric morbidity in military veterans who have served since September 11, 2001. Journal of Psychiatric Research 43 $830-836$.

Deprince, A.P Chu, A, 2008. Perceived benefits in trauma research: examining methodological and individual difference factors in responses to research participation. Journal of Empirical Research on Human Research Ethics 3, 35-47.

DePrince, A.P., Freyd, J.J., 2004. Costs and benefits to research participants of being asked about trauma history. Journal of Trauma Practice 42, 23-25.

Ferrier-Auerbach, A.G., Erbes, C.R., Polusny, M.A., 2009. Does trauma survey research cause more distress than other types of survey research? Journal of Traumatic Stress 22, 320-323.

First, M.B.S., Gibbon, M., Williams, J.B.W, 1997. Structured Clinical Interview for DSM-IV for Axis I Disorders (SCID). Biometrics Research, New York State Psychiatric Institute, New York, NY.

Gerin, W., Ogedegbe, G., Schwartz, J.E., Chaplin, W.F., Goyal, T., Clemow, L. Davidson, K.W., Burg, M., Lipsky, S., Kentor, R., Jhalani, J., Shimbo, D., Pickering T.G., 2006. Assessment of the white-coat effect. Journal of Hypertension 24 67-74.

Griffin, M.G., Resick, P.A., Waldrop, A.E., Mechanic, M.B., 2003. Participation in trauma research: Is there evidence of harm? Journal of Traumatic Stress 16, 221-227.

Grubaugh, A.L., Tuerk, P.W., Egede, L.E., Frueh, B.C., 2012. Perceptions of PTSD research participation among patients with severe mental illness. Psychiatry Research 200, 1071-1073.

Newman, E, Kaloupek, D., 2009. Overview of research addressing ethical dimensions of participation in traumatic stress studies: autonomy and beneficence. Journal of Traumatic Stress 22, 595-602.

Parslow, R.A., Jorm, A.F., O'Toole, B.I., Marshall, R.P., Grayson, D.A., 2000. Distress experienced by participants during an epidemiological survey of posttraumatic stress disorder. Journal of Traumatic Stress 13, 465-471.

Resick, P.A., Iverson, K.M., Artz, C.E., 2009. Participant reactions to a pretreatment research assessment during a treatment outcome study for PTSD. Journal of Traumatic Stress 22, 316-319.

Reynolds, S.K., Lindenboim, N., Comtois, K.A., Murray, A., Linehan, M.M., 2006. Risky assessments: participant suicidality and distress associated with research assessments in a treatment study of suicidal behavior. Suicide and LifeThreatening Behavior 36, 19-34.

Saunders, J.B., Aasland, O.G., Babor, T.F., de la Fuente, J.R., Grant, M., 1993 Development of the alcohol use disorders identification test (audit): who collaborative project on early detection of persons with harmful alcohol consumption-ii. Addiction 88, 791-804.

Scarvalone, P.A., Cloitre, M., Spielman, L.A., Jacobsberg, L., Fishman, B., Perry, S.W. 1996. Distress reduction during the structured clinical interview for dsm-iii-r. Psychiatry Research 59, 245-249.

Solomon, Z, Mikulincer, M., 2006. Trajectories of PTSD: a 20-year longitudina study. American Journal of Psychiatry 163, 659-666.

Walker, E.A., Newman, E., Koss, M., Bernstein, D., 1997. Does the study of victimization revictimize the victims? General Hospital Psychiatry 19, 403-406. 